\title{
Breathing, sleep state, and rectal temperature oscillations
}

David M Tappin, Rodney P K Ford, Kerrie P Nelson, Brent Price, Paul M Macey, Richard Dove, Janine Larkin, Beryl Slade
Community Paediatric Unit, HealthLink South, PO Box 1475, Christchurch, New Zealand

D M Tappin

R P K Ford

K P Nelson

B Slade

J Larkin

Department of

Medical Physics and Bioengineering,

Christchurch Hospital, Christchurch, New

Zealand

B Price

$\mathbf{R}$ Dove

Department of

Electrical and

Electronic

Engineering,

University of

Canterbury,

Christchurch, New

Zealand

P M Macey

Correspondence to:

Dr D M Tappin

Department of Child Health,

Royal Hospital for Sick

Children, Yorkhill, Glasgow G3 8SJ.

Accepted 30 January 1996

\begin{abstract}
Overheating may cause terminal apnoea and cot death. Rectal temperature and breathing patterns were examined in normal infants at home during the first 6 months of life. Twenty one infants had continuous overnight rectal temperature and breathing recordings for 429 nights (mean 20.4 nights, range 7-30) spaced over the first six months of life. Periods when breathing was 'regular' were directly marked on single night records. Sleep state was determined from respiratory variables. 'Regular' breathing was a reliable marker of 'quiet' sleep (specificity $93 \%$ ). The duration of 'quiet' sleep increased from 6 to 22 minutes from two weeks to three months of age and then remained static, as did the proportion of sleep spent in the quiet phase $(9 \%$ to $34 \%)$. Rectal temperature fell during $66 \%$ of quiet sleep and usually rose during rapid eye movement (REM) sleep. The drop in rectal temperature was maximal at the start of quiet sleep, whereas the maximum rise during REM sleep was reached after 10 to 15 minutes. Oscillations in rectal temperature are associated with changes in sleep and breathing state. The maturation of rectal temperature patterns during the first six months of life are closely related to a maturation of sleep state and breathing patterns.

(Arch Dis Child 1996; 74: 427-431)
\end{abstract}

Keywords: body temperature, respiration, sleep stages, sudden infant death.

There is evidence of a link between the sudden infant death syndrome (SIDS or cot death) and overwrapping or raised environmental temperature. ${ }^{1}$ Infant thermoregulation and respiratory control are closely linked and there are some hypotheses as to how this link may lead to cot death.

Infant thermoregulation is related to core temperature, in that failure of thermoregulation will lead to an abnormally high or low core temperature. We have described a regular oscillation of rectal temperature in overnight recordings of infants who have previously experienced an apparent life threatening event (ALTE). ${ }^{2}$

This work has now been extended to record temperature and breathing of normal infants in their home environment during the first six months of life, when $80 \%$ of cot deaths occur. In this paper we explore the relations between sleep state, breathing patterns, and oscillations of rectal temperature.

\section{Methods}

ENROLMENT

Ethics committee approval allowed continuous overnight temperature and respiratory patterns to be recorded on infants at home. Recruiting of normal infants was made through newspaper articles asking women in late pregnancy to volunteer their infants for temperature and respiration recording during the first six months after birth. We specified non-smoking mothers with full term deliveries and no neonatal problems. The study research nurse explained the study and demonstrated the HomeLog ${ }^{3}$ equipment and recording procedures in the home. Parents were then asked to phone her once their baby was born if they still wanted to join the study. At the first recording at two weeks of age the research nurse delivered the equipment and asked for written consent. She was available by telephone in case of equipment breakage or other problems.

\section{PATIENTS}

Between 1 September 1993 and the 31 August 1994, 21 infants had at total of 429 (mean 20.4, range 7 to 30 ) nights of data recorded, using four HomeLog systems. ${ }^{3}$ All infants (12 male and nine female) were born at term and had no neonatal problems. Mean birth weight was $3850 \mathrm{~g}$ (range 3000 to 4300). Twenty infants were breast fed. Twenty infants were put down to sleep on their back or side, one infant was placed prone to sleep. No mothers smoked during or after pregnancy. Twenty infants thrived throughout their first six months; the other infant, who started on the 90 th weight centile, fell to the 10th at three months, in association with failure of lactation; he started formula feeds and soon returned to the 75 th centile. Each infant was recorded over four predetermined periods during the first six months of life: 2-3 weeks of age for three nights; at the first vaccination at 6 weeks for seven nights (three nights before the vaccination, the night of the vaccination, and three nights afterwards); and similarly for seven nights at each of the routine vaccinations at 3 and 5 months of age. One infant had only a 5-month recording, two had a 3-month and a 5-month recording, three had a 6-week, a 3-month, and a 5-month recording, and 15 had a complete set. 
EQUIPMENT

Each HomeLog system was based on a 286 IBM-PC compatible laptop computer with custom hardware and software allowing up to 16 signals to be recorded simultaneously to the hard disc. ${ }^{3}$ Temperature probes were based on a semiconductor and were validated locally. ${ }^{4}$ The probes had a relative accuracy of $0.01^{\circ} \mathrm{C}^{4}$; thus (within record) temperature changes were measured very accurately. Regular calibration was performed on each set of temperature probes to an absolute accuracy of $0 \cdot 1^{\circ} \mathrm{C}$ using a mercury in glass reference thermometer. Temperature measurements were sampled at a rate of $1 \mathrm{~Hz}$, digitised, and recorded. Rectal temperature was recorded from a probe $5 \mathrm{~cm}$ from the anal margin, sealed in a soft plastic feeding tube. A second probe in this plastic tube $2 \mathrm{~cm}$ from the anal margin was also present. If similar recordings were present from both the rectal and anal probes, then the rectal probe was positioned correctly and had not slipped down. Graseby MR10 respiration monitors were used to record breathing by abdominal movement. This signal was sampled at $10 \mathrm{~Hz}$. Monitors were calibrated monthly to reduce troublesome false alarms. Parents were supplied with a clipboard to log nightly details which included: the time the baby was put down to sleep, sleep times, time of morning waking, and the start and finish of feeds and nappy changes. A digital clock attached to the computer, used by parents for noting times, was synchronised with the HomeLog computer clock. After each three or seven night period of recording, data - about 20 megabytes - were downloaded from the HomeLog PC to an optical drive, allowing immediate access for analysis and graphing.

\section{ANALYSIS}

Records were inspected using BabyLog software ${ }^{5}$ and bad segments removed. Bad segments (areas of recording that had data loss of either temperature or respiration) were common in the early stages of the study when temperature probes were prone to failure. Improved design of the wiring of the temperature sensors solved these problems. Graseby monitors were also prone to false alarms, which sometimes led parents to disconnect them. This was resolved by monthly calibration. Other bad sectors removed included periods when anal temperature was not closely related to rectal temperature, indicating that the probe may have been dislodged.

Information from parental logs was transferred onto the computer record. Midday was defined as the start of each 'day', so that one whole night of recording would be in each 'day'. Further variables marked were: periods of sleep (' $S$ ' in fig 1; this particular sleep period was continuous, although many were interrupted by feeds or nappy changes); periods of 'regular' breathing (' $Q$ ' in fig 1 , marked manually by visual inspection as the low amplitude areas seen on a complete night of Graseby signal); feed and nappy changes, taken from the parental logs (no feeds or nappy changes occurred in this night of recording); and the initial drop in rectal temperature on falling asleep (' $O$ ' in fig 1 , marked by visual inspection of the rectal temperature trace). Figure 2 shows the same night of Graseby signal. The arrows point to expanded $30 \mathrm{~s}$ periods of this Graseby signal. The high amplitude area can be seen as 'active' breathing, with highly variable breath lengths, the low amplitude area can be seen as 'regular' breathing, with stable breath lengths.

BabyLog analysis used peak to peak detection to calculate the length of each individual breath. ${ }^{6}$

For this analysis we chose to cut each record into sequential $5 \mathrm{~min}$ epochs. Although $1 \mathrm{~min}$ epochs have used for sleep staging using respiratory variables, ${ }^{7}$ we were also interested in temperature changes. The oscillations in rectal temperature are of the order of $1 \mathrm{~h},{ }^{2}$ so a $5 \mathrm{~min}$ epoch was considered an adequate period to detect changes in temperature but short enough for sleep staging to be accurate. In each epoch there were 300 rectal temperature readings from which were derived the median rectal temperature and the rate of change in rectal temperature in each epoch. The median rather than the mean was used to minimise the effect of outlying points in case of artefacts. Also for each epoch, breath lengths (usually 150 to 200 breaths for each $5 \mathrm{~min}$ epoch) were calculated and transformed into median breath length (breath length $=60 /$ breath rate), upper quartile breath length, and lower quartile breath length. The differences between the latter two have been used extensively to describe breath rate variability, an important variable used to define sleep state. ${ }^{7}$ The data were imported into the SAS statistical package ${ }^{8}$ and comparisons made between multiple variables.

Each night record was visibly examined for the presence of obvious regular oscillations in rectal temperature. ${ }^{2}$ Oscillations were defined as of at least $0 \cdot 1^{\circ} \mathrm{C}$ in amplitude and of about $1 \mathrm{~h}$ in duration and could be seen and were obvious to the naked eye throughout at least $50 \%$ of the night trace (fig 1).

Sleep state (awake, rapid eye movement [REM], quiet, or undetermined) was assigned to each epoch using respiratory variables according to the method described by Harper. ${ }^{7}$ 'Harper' sleep state classification was made for $83.7 \%(n=11420)$ of 13646 epochs, with $16.3 \%(n=2226)$ 'undetermined': awake $1 \cdot 8 \%$ $(n=250)$, REM sleep $55 \cdot 7 \%(n=7602)$, and quiet sleep $26 \cdot 2 \%(n=3568)$. The length of each period of quiet and REM sleep (in epochs) was defined by examining sequential epochs recorded on each infant, the position of each epoch within the period of REM or quiet sleep was also defined. The calculated quiet sleep state epochs have been entered as ' $F$ ' in fig 1 (between ' $Q$ ' and ' $O$ '). From fig 1, 'regular' breathing periods (' $Q$ '), closely approximate to quiet sleep (' $F$ ').

For this report we have analysed the control nights only: the three nights of recording at two weeks of age $(n=43)$, and the three nights prior to vaccination at six weeks $(n=44)$, three months $(n=55)$, and five months $(n=49)$.

The $95 \%$ confidence intervals around the 


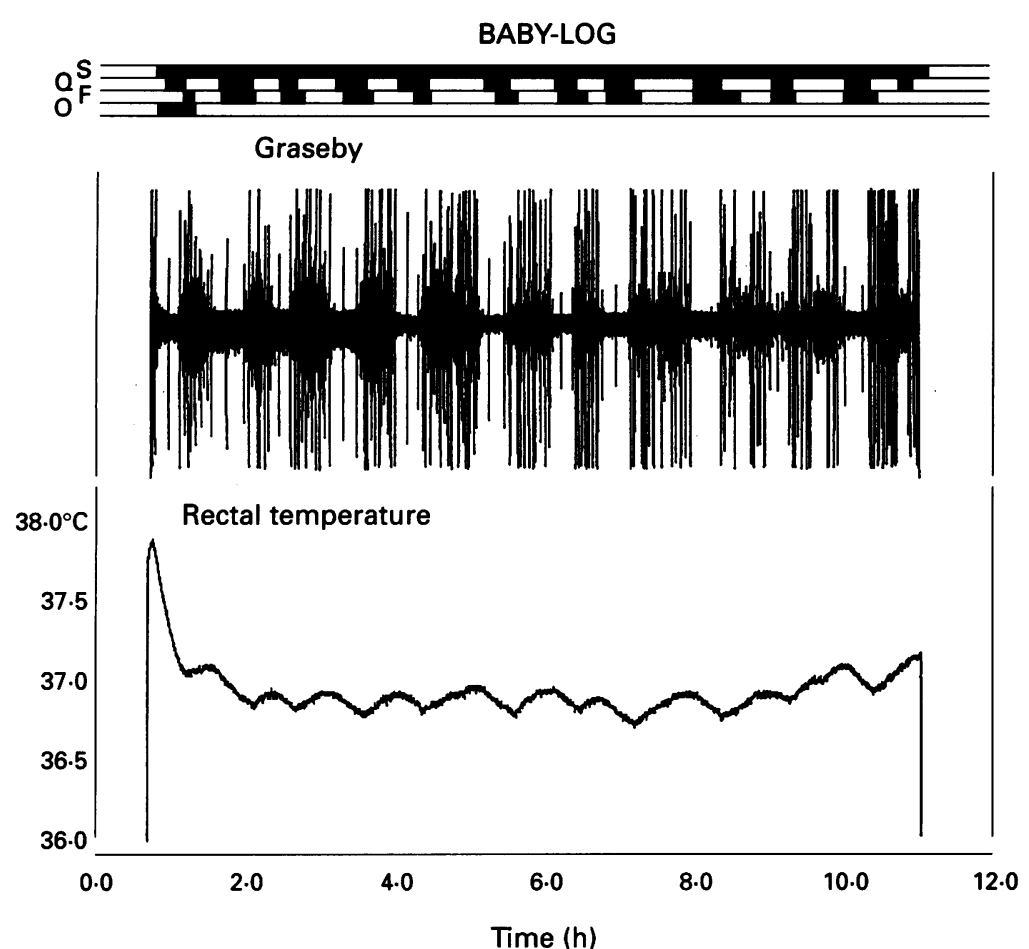

Figure 1 An 11 h overnight recording in a 3 month old well infant. The rectal temperature shows regular oscillations with amplitude of $0 \cdot 2^{\circ} \mathrm{C}$ and period of $1 \mathrm{~h}$. The Graseby respiration signal shows a pattern 'regular' and 'active' breathing with the same periodicity of about $1 \mathrm{~h}$. The key to the scale below the title 'BABYLOG' shows $S=$ periods of sleep (this child was asleep throughout the record), $Q=$ 'regular' breathing, determined by visual inspection, $F=$ the epochs designated by Harpers method as 'quiet' sleep, $0=$ the region of temperature drop associated with the onset of sleep.

mean drop in rectal temperature were calculated by adding and subtracting $1.96 \times$ standard error $(\mathrm{SE})$ of the mean. $\mathrm{SE}=$ the standard deviation for each mean value divided by the square root of the number of values which made up that mean.

A total of 13646 control night epochs (1137 hours) which had adequate temperature and respiratory signals was available for analysis. This dataset was used to describe the relations

Breathing patterns

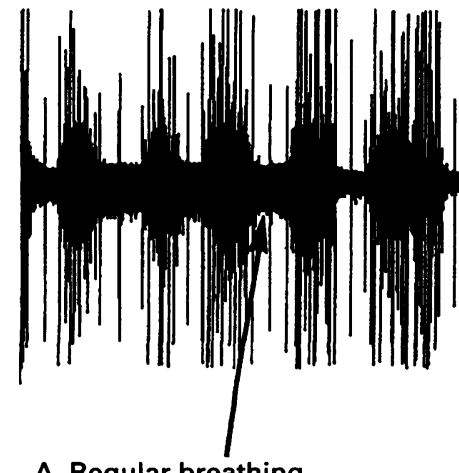

A. Regular breathing

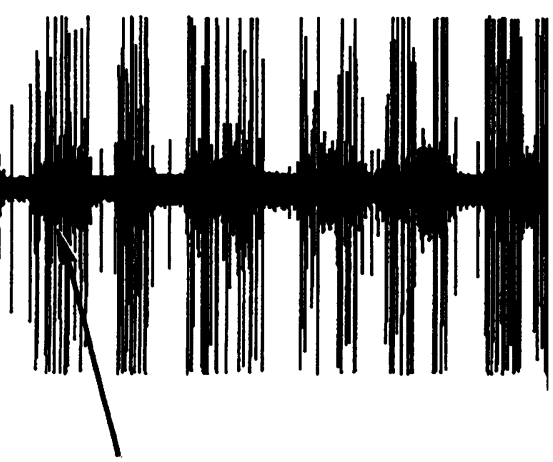

B. Active breathing

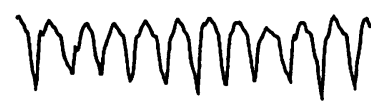

$30 \mathrm{~s}$

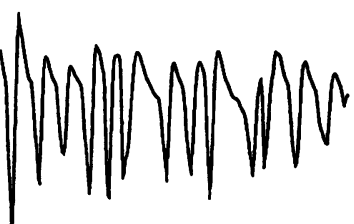

Figure 2 The same night of Graseby recording is shown as fig 1. Arrows point to areas where the Graseby recording has been expanded to show two 30 s periods, $A$ and $B$. $A$ is a period marked as 'regular'breathing on the whole night record: the expanded trace shows a low amplitude waveform, but more importantly breaths of equal length. $B$ is a period 'active' breathing, with a higher amplitude waveform, but with breaths of variable length. between oscillations in rectal temperature, 'regular' breathing observed in overnight records (figs 1 and 2), and sleep state in normal infants at home during the first six months of life.

\section{Results}

In $70 \%$ of control night records, regular oscillations in rectal temperature could clearly be seen: 21 of 43 nights (49\%) at two weeks, 32 of 44 nights (73\%) at six weeks, 37 of 55 nights $(68 \%)$ at three months, and 44 of 49 nights $(90 \%)$ at five months. A correlation was seen between increasing infant age and the presence of visible oscillations in rectal temperature $\left(R^{2}=0 \cdot 78\right)$.

On inspection, the oscillations in rectal temperature were temporally associated with a change in respiratory pattern (fig 1). As breathing patterns are related to sleep state, ${ }^{7}$ we first examined if 'regular' breathing (as identified on our recordings) was related to sleep state (as defined by Harper's method). Regular breathing accounted for $28.9 \%$ of all epochs, while Harper's 'quiet' sleep accounted for $26 \cdot 2 \%$. There were 3568 epochs of Harper quiet sleep in all records, and 2495 were marked as regular breathing. There were 7602 epochs of Harper REM sleep and 7039 were not marked as regular breathing. Regular breathing had $70 \%$ sensitivity and $93 \%$ specificity when used to describe quiet sleep defined by Harper's method. Figure 1 illustrates the close association between regular breathing (' $Q$ ') and Harper quiet sleep (' $F$ '), which has been marked on this computer record. Harper sleep state was used for subsequent analysis, because of the close association with breathing state.

The relation between rectal temperature changes and sleep state for each epoch is presented in table 1A. To ensure that these results were not due to spurious findings we sequentially removed epochs which may have masked the close association between sleep state and temperature change. Firstly, all epochs which parents had recorded as non-sleep were removed, then epochs without visible oscillations in rectal temperature were removed, then epochs associated with the initial drop in rectal temperature on falling asleep were removed. Table 1B shows the temperature changes in the remaining 7918 epochs. By this progressive analysis, portions of data were removed which might have influenced the direct association

Table 1 Percentage of epochs with temperature changes in each 'Harper' sleep state category

\begin{tabular}{llcc}
\hline & \multicolumn{3}{c}{ Rectal temperature changes (\%) } \\
\cline { 2 - 4 } Harper sleep state & Fall & Static & Rise \\
\hline$A(n=13646)$ & & & \\
Quiet $(n=3568)$ & 66 & 14 & 20 \\
REM $(n=7602)$ & 35 & 11 & 54 \\
Awake $(n=250)$ & 35 & 8 & 57 \\
Undetermined $(n=2226)$ & 52 & 14 & 34 \\
$B(n=7918)$ & & & \\
Quiet (n=2604) & 66 & 14 & 20 \\
REM (n=3857) & 24 & 12 & 64 \\
Awake (n=81) & 10 & 12 & 78 \\
Undetermined (n=1376) & 64 & 12 & 24 \\
\hline
\end{tabular}

REM=rapid eye movement. 
Table 2 Length of periods of 'quiet' and rapid eye movement (REM) sleep and infant age

\begin{tabular}{llllll}
\hline \multirow{2}{*}{$\begin{array}{l}\text { Harper sleep } \\
\text { state }\end{array}$} & \multicolumn{4}{l}{ Mean length of Harper sleep state periods (minutes) by age } \\
\cline { 2 - 6 } & 2 Weeks & 6 Weeks & 3 Months & 5 Months & Overall \\
\hline Quiet (SD) & $10 \cdot 3(6 \cdot 4)$ & $17 \cdot 6(8 \cdot 5)$ & $22 \cdot 1(10 \cdot 7)$ & $20 \cdot 0(11 \cdot 0)$ & $18 \cdot 9(10 \cdot 6)$ \\
\% Time & 9 & 26 & 34 & 30 & 26 \\
REM (SD) & $54 \cdot 4(39 \cdot 5)$ & $31 \cdot 4(21 \cdot 4)$ & $23 \cdot 0(15 \cdot 5)$ & $20 \cdot 2(13 \cdot 0)$ & $29 \cdot 1(24 \cdot 7)$ \\
\% Time & 80 & 61 & 46 & 43 & 56 \\
\hline
\end{tabular}

between quiet sleep and a fall in rectal temperature, which is demonstrated in fig 1 . The association between sleep state and change in rectal temperature was remarkably stable.

The length of sleep state periods is illustrated in table 2 . The length of quiet sleep periods increased, and REM sleep periods decreased, as infants got older. The overall proportion of time spent in the two sleep states changed in a similar way. At two weeks of age only $9 \%$ of the epochs were spent in Harper quiet sleep, whereas $80 \%$ were spent in REM sleep; at six weeks, $26 \%$ were spent in quiet sleep and $61 \%$ in REM sleep; at three months, $34 \%$ were spent in quiet sleep and $46 \%$ in REM sleep; and at five months, $30 \%$ were spent in quiet sleep and $43 \%$ in REM sleep.

The mean temperature change in epochs of quiet and REM sleep are illustrated in fig 3, with $95 \%$ confidence intervals. Each period of REM or quiet sleep was made up of a number of sequential epochs. These epochs were numbered from ' 1 ', the first epoch in a period of sleep, to ' $n$ ', the total number of epochs which made up the period of sleep. It can be seen that the rate of fall of temperature within quiet sleep was on average higher than the rate of rise in REM sleep. The fall in temperature had usually started before the first epoch of quiet sleep, and was maximal during it. The rise in temperature during REM sleep was on average maximal during the third epoch of REM sleep.

\section{Discussion}

The aim of this study was to look at the respiratory and temperature patterns of infants at low risk of cot death. Many studies have

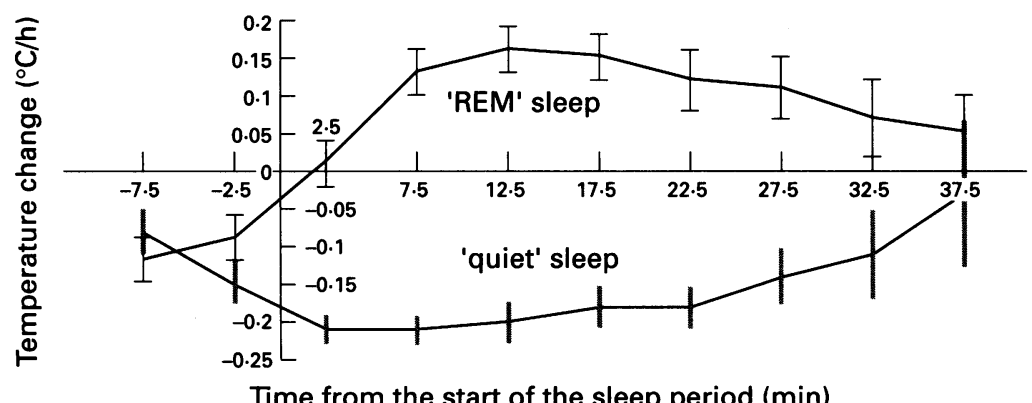

Figure 3 All periods of rapid eye movement (REM) and 'quiet' sleep are illustrated together in this figure. The total temperature change over all the first epochs within periods of REM sleep were added and then divided by the number of first REM epochs to give a mean change of $+0.01^{\circ} \mathrm{C}$ per hour. This value was marked at 2.5 min after the start of mean change of $+0.01^{\circ} \mathrm{C}$ per hour. This value was marked at $2 \cdot 5$ min after the start of temperature change over all first epochs was divided by the number of epochs to give a mean change of $-0.21^{\circ} \mathrm{C}$ per hour. Similar calculations were made for all second epochs and so on. The error bars indicate that we are $95 \%$ confident that the mean value of temperature change was within this range, and was calculated as: the mean + or $-1.96 \times$ SEM. The length of the error bars largely inversely reflected the number of epochs contributing towards the mean value. For REM sleep, there were 1249 'first' epochs and 253 'eighth' epochs. For 'quiet' sleep there were 939 'first' epochs and 27 'eighth' epochs. looked at high risk infants; data on such infants in Christchurch have shown the relations between respiratory patterns and rectal temperature oscillations. ${ }^{2}$ The present report describes this association for low risk infants in more detail on a large dataset of 1137 hours of recording from 21 normal, well infants during their first six months of life. We have clearly shown that there is a strong relation between rectal temperature and breathing and sleep state. During epochs of quiet sleep (or 'regular' breathing) the rectal temperature fell. Conversely during REM sleep there was an associated rise in rectal temperature.

A random sample of normal babies was not possible to enrol because the ethics committee would not allow the parents to be approached directly. Data collection was only logistically possible on 20 babies at each age so it was important to look at a homogeneous sample of infants. We looked at healthy babies at low risk of cot death over a full six month period so that comparison could be made with data already collected on high risk infants.

The BabyLog methods allowed physiological variables to be displayed and analysed and information from parental logs could be marked on the computer record. So that rectal temperature and respiratory patterns could be compared, all records were cut into sequential five minute segments or epochs. By the use of respiratory variables, Harper $^{7}$ was able to assign sleep state to infants from one week to six months with $80 \%$ accuracy. This compared very favourably with trained observers using EEG, EOG, and other somatic variables, who only achieved $85 \%$ accuracy. ${ }^{7}$ We used the Harper method to assign sleep state using breath rate and breath rate variability for each epoch in the SAS dataset. Harper was able to assign sleep state to $95 \%$ of one minute epochs. We were able to assign sleep state to $83.7 \%$ of five minute epochs. This difference may be explained by the epoch length. As one complete period of oscillation in breathing and sleep takes about an hour, two epochs per hour will be likely to span from quiet to REM sleep or vice versa. With one minute epochs, only two minutes will be affected out of each hour, which is $3.3 \%$ of epochs. But if epochs are five minutes in length, then up to 10 minutes of each hour will be affected (up to $17 \%$ of epochs). The five minute epoch time was chosen to allow detectable changes in temperature and breathing to occur but to be short enough to allow accurate sleep staging.

There was a close association between quiet sleep and regular breathing. Regular breathing was only marked when it was definite, and periods of possible regular breathing were excluded; therefore the $70 \%$ sensitivity of regular breathing at picking up all quiet sleep periods is reasonable. The $93 \%$ specificity of regular breathing at correctly describing quiet sleep is good. Regular breathing, defined by looking at a full night of Graseby signal and picking those areas with an even, low amplitude breathing signal, can therefore be used as a good approximation of quiet sleep state.

When we further examined the relation 
between sleep state and temperature oscillations (which is well shown in fig 1) by removing other causes of a fall in temperature, non-sleep periods, and nights without apparent oscillations, the proportion of quiet sleep epochs with falls in rectal temperature did not change (table 1). This can be explained by the presence of a phase difference (or time lag) between the onset of quiet sleep and the fall in rectal temperature. In these 21 normal infants, the temperature drop had on average started 10 minutes before the onset of quiet sleep and the fall was maximal (fig 3) during the first epoch of quiet sleep. This contrasted with the rise in temperature associated with REM sleep which on average began at the start of the REM sleep period but did not reach its peak for 10-15 minutes.

This dataset shows an increase in length of quiet sleep periods and an increase in the proportion of epochs spent in quiet sleep from the age of 2 weeks $(9 \%)$ to the age of 3 months (34\%). Temperature fall occurs during quiet sleep. We therefore hypothesise that the maturation of infant temperature pattern described by Wailoo and Petersen ${ }^{9}$ is closely associated with as similar maturation of sleep state involving an increase in the proportion of time spent in quiet sleep.

We have now clearly established that rectal temperature rise and fall is associated with changes in breathing and sleep state. The oscillations in rectal temperature must now be considered to involve changes in sleep state and breathing pattern. The presence or absence of temperature oscillations under various circumstances such as infant age, illness, stress, and overwrapping, may be a significant developmental marker for the maturation of respiration/sleep/temperature control mechanisms, and thus play a part in explaining the enigma of cot death.

The Canterbury Cot Death Fellowship supported DMT; the Scottish Cot Death Trust supported BS (research nurse), NCHRF (The Cot Death Association) supported KN (biostatistician). We wish to thank the parents who participated.

1 Fleming PJ, Levine MR, Azaz Y, Wigfield R, Stewart AJ. Interactions between thermoregulation and the control of respiration in infants: possible relationship to sudden infant death. Acta Paediatr Suppl 1993; 389; 57-9.

2 Brown PJ, Dove RA, Tuffnell CS, Ford RP. Oscillations of body temperature at night. Arch Dis Child 1992; 67: body tem-8.

3 Ford RP, Brown PJ, Dove RA, Tuffnell CS, Macey PM. Ford RP, Brown PJ, Dove RA, Tuffnell CS, Macey PM.
HomeLog: long-term recording of infant temperature, respiratory and cardiac signals in the home environment. f Paediatr Child Health 1992; 28 (suppl 1): S26-32.

4 Brown J, Dove R, Price B, Fong S, Ford R. Continuous multiple location body temperature measurement on infants. Aust Phys Eng Sci Med 1990; 13: 85-7.

5 Dove R, Brown J, Fright R, Tuffnell C, Ford R. Computer polygraphic system for infants at risk for sudden infant death syndrome (SIDS). Aust Phys Eng Sci Med 1990; 13: 188-91.

6 Macey PM, Ford RPK, Brown PJ, Larkin J, Fright WR, Garden KL. Apnoea detection: human performance and reliability of a computer algorithm. Acta Paediatr 1995; 84: 1103-7.

7 Harper RM, Schechtman VL, Kluge KA. Machine classification of infant sleep state using cardiorespiratory measures. Electroencephalogr Clin Neurophysiol 1987; 67: 379-87.

8 SAS Institute Inc, Cary, NC.

9 Lodemore M, Petersen SA, Wailoo MP. Development of night time temperature rhythms over the first six months of life. Arch Dis Child 1991; 66: 521-4. 